\title{
Deep Exploration of Bifidobacteria through Metabolomics Study
}

\author{
Juan Li1,2, Yatao Jiang1, Yihao Shen', Qingzhi Li ${ }^{1}$, Zhongke Sun ${ }^{{ }^{*}}$ \\ ${ }^{1}$ Institute of Food and Drug Inspection, College of Life Sciences and Agronomy, Zhoukou Normal University, Zhoukou, China \\ ${ }^{2}$ Library of Zhoukou Normal University, Zhoukou, China \\ Email: *sunzh@daad-alumni.de
}

How to cite this paper: Li, J., Jiang, Y.T., Shen, Y.H., Li, Q.Z. and Sun, Z.K. (2018) Deep Exploration of Bifidobacteria through Metabolomics Study. Journal of Biosciences and Medicines, 6, 57-62. https://doi.org/10.4236/jbm.2018.65008

Received: April 1, 2018

Accepted: May 20, 2018

Published: May 23, 2018

Copyright $\odot 2018$ by authors and Scientific Research Publishing Inc. This work is licensed under the Creative Commons Attribution International License (CC BY 4.0).

http://creativecommons.org/licenses/by/4.0/

(c) (i) Open Access

\begin{abstract}
Bifidobacteria are probiotic bacteria with multiple health-promoting properties for human being. The global market for probiotics, especially for bifidobacteria is booming. However, the entire market is still at an early stage as there is nearly no fine products developed yet except the whole bacterial cells. The maturation of metabolomics technologies make it possible to study complex mixture with high-throughput, comprehensive maps and libraries. Therefore, we prospect that metabolomics studies mainly based on liquid/gas chromatography-mass spectrometry (LC/GC-MS) can deepen our understanding in detail during the study of metabolic mechanisms of bifidobacteria. These studies can be conducted at three phases, including non-targeted, targeted metabolomic analysis of bifidobacteria, and specific metabolites production through metabolic engineering and fermentation. Metabolomic studies of bifidobacteria will allow us to fully explore their metabolic mechanisms and to utilize metabolites that contribute to human health. In particular, bifidobacteria derived conjugated linoleic acids and bacteriocins are two kinds of fined products that may have great potentials in the future and can be used as food additives.
\end{abstract}

\section{Keywords}

Bifidobacteria, Metabolomics, Metabolites, Conjugated Linoleic Acid, Probiotics Derived Fine Products

\section{Bifidobacteria Are Probiotic Bacteria with Multiple Health-Promoting Properties}

Bifidobacteria are the most abundant and most important physiological bacteria inhabiting the intestines of humans and animals. As the core subject of micro- 
ecological research, bifidobacteria exert their biological functions through barrier, nutrition, immunity, anti-tumor, control endotoxemia, anti-aging, anti-tumor and other physiological effects [1] [2]. The number of bifidobacteria in the human intestine is an indicator to check whether a person is healthy. Studies have shown that Bifidobacterium adheres to intestinal epithelial cells; forms specific microecological circles with other related bacteria; produces specific ecological effects; and inhibits colonization and translocation of intestinal pathogenic bacteria and conditional pathogenic bacteria [3]. Bifidobacteria may also bind to intestinal epithelial cells competitively by synthesizing certain metabolites or certain proteins to antagonize the pathogenic bacteria and form a barrier against infection [4]. Therefore, Bifidobacterium can be used for counterbalance the dysbiosis caused by the abuse of antibiotics, radiotherapy, chemotherapy and other factors. At present, in addition to being added to health products, the clinical application of Bifidobacterium extends from the department of gastroenterology to the departments of dentistry, dermatology, oncology, and neurology [5] [6].

\section{The Global Probiotic Market Is Booming}

Probiotics are an important concept for health care in the 21st century. As an emerging component of new generation pharmaceutical products and dietary supplements, probiotics have become a large business in the international market. In 2014, the global probiotic market has outreached US\$60 billion

(https://www.transparencymarketresearch.com/probiotics-market.html). The concept that probiotics are beneficial to human health and consumers' growing interest in preventive healthcare are main factors for the development of the market. As people's understanding of health increases, prophylactic drugs containing probiotics as their active ingredient are gradually appealing. In Asia and Europe, probiotics are widely used as health foods and medicines. In the global probiotic market, the European market is the largest and the fastest growing with an average annual growth rate of around $20 \%$

(https://www.grandviewresearch.com/industry-analysis/probiotics-market). Although the probiotic market has made considerable progress in recent years, the entire market is still at an early stage of development.

\section{Metabolomics Studies Accelerates Deep Investigation}

Modern scientific research has changed from traditional single-gene research to functional related research. This is due to the complexity of the organism and, on the other hand, benefited from the improvement of technology. The maturation of various omics-technologies makes it possible to study complex mixture with high-throughput, comprehensive maps and libraries [7]. Especially, the maturation of metabolomics techniques that based on liquid/gas chromatography mass spectrometry (LC/GC-MS) can deepen our understanding in detail during the study of molecular metabolic mechanisms of bifidobacteria. Metabo- 
lomics studies of bifidobacteria will allow us to fully explore the physiological and metabolic mechanisms of bifidobacteria and analyze metabolites that contribute to human health [8] [9]. As most of these metabolites have not been fully analyzed, we still have insufficient evidence for many of the probiotic mechanisms of bifidobacteria, which is a major factor slowing the development of probiotics derived fine products.

\section{Bifidobacteria Have Many Uncharacterized Metabolites}

In addition to lactic acid and acetic acid, various metabolites of bifidobacteria contribute to the probiotic functions demonstrated after application of their host [10]. One class of these metabolites is bioactive fatty acids, such as Conjugated Linoleic Acid (CLA), which includes a series of positional and geometrical isomers of linoleic acid with double bonds at the carbon 9.11 or 10.12 positions. CLA is almost an indispensable nutrient for modern people [11]. It can enhance the fluidity of cell membranes, prevent the proliferation of vascular cortex, and maintain the normal function of organ microcirculation. CLA can also clear the garbage in the blood vessels, effectively regulate blood viscosity, achieve vasodilation, smooth blood pressure; also regulate tumor necrosis factor, interleukin, prostaglandin or nitrogen oxides, while reducing allergic immune response. Another class with particular interest is bacteriocin, which is a class of polypeptides or precursor polypeptides with antibacterial activity that exhibits a narrow inhibitory spectrum for the same species of closely related strains [12]. Due to the unique mode of action as well as the rapid expansion of multi-drug resistance, it is considered that bacteriocin will be the most powerful alternative to antibiotics in the future.

\section{Metabolomics Study of Bifidobacteria}

\subsection{Phage I: Non-Targeted Metabolomics Analysis of Bifidobacteria}

Non-targeted metabolomics involves comparing the metabolomes of the control and experimental groups (all metabolites of a given organism) to find differences in their metabolites. The analysis typically includes: 1) Metabolic Profile Analysis: in a set of experimental and control samples, the metabolites of interest with statistically significant changes in abundance were sought. 2) Identification: after the metabolic profile analysis, the chemical structures of these metabolites were determined. 3) Interpretation: explaining the relationship between discovered metabolites and biological processes or biological states. Non-targeted methods are mainly used for the study of metabolic phenotyping or differential metabolite discovery. As an analytical technique, non-targeted metabolomics can qualitative determine and relative quantify metabolites in biological systems as much as possible, which maximizes the total metabolite information. The flexibility and universality of LC-MS technology made it the most commonly used technology in metabolomics research [13]. We conducted LC-MS for comprehensive study of metabolites that produced by three strains of Bifidobacterium 
fermented for 48 hours in MRS medium and found many useful metabolites that have important functions for human. However, there are still many problems to be solved in LC-MS-based metabolomics analysis. For example, many trace metabolites have important physiological functions, but are difficult to detect [14]. Thus, simultaneous use of GC-MS is necessary for bifidobacterial metabolome study to complement the deficiencies of the single technique.

\subsection{Phase II: Targeted Metabolomic Analysis of Bifidobacteria}

Targeted metabolomics analysis is used for highly sensitive detection and accurate quantitative analysis of certain metabolic pathways or known metabolites of interest. It is mainly used for the verification of certain differential metabolites and other classic targeted metabolomics. For such study, LC-MS analysis generates a selective reaction monitoring ion pair from the specific standards at first, and then performs a second analysis of the target metabolite in the sample. Based on a non-targeted comprehensive scan, we established a metabolite database for bifidobacteria. Through preliminary comparisons, it is possible to determine which components are specific or common metabolites in bifidobacteria. In particular, targeted analysis of lipids, bacteriocins, functional oligosaccharides, and absolute quantification of them should be performed [15]. This can be done in two ways. One is comparing the content of these metabolites in different media, and the other is changing the content in different culture time (growth phase) in the same medium [16]. Through these analyses, biochemical characteristics of some metabolites with high-value can be determined during production, e.g. CLAs and vitamins. We conducted targeted metabolic studies for CLAs produced by above three strains of Bifidobacterium fermented for 48 hours in different media and found species/strains specific production patterns on the composition and amount.

\subsection{Phase III: Produce Metabolites through Metabolic Engineering and Fermentation}

The formation of metabolites is inseparable from the fermentation process, and metabolic engineering is the main way to change metabolic flux and control the formation of metabolites [17]. The use of multi-gene metabolic engineering, together with gene regulation, metabolic regulation, and biochemical engineering, can create new pathways to produce specific products [18]. Through the metabolic network analysis of the target metabolites obtained during phase II, the enzyme and substrate can be determined and they can be utilized for adjustment from two aspects [19]. At the same time, the formation of metabolites is closely related to the fermentation [20]. Therefore, optimization the production of target metabolites from the aspects of the culture media and the fermentation parameters is required. Through these experiments, industrial production processes and technologies can be established, which are of great significance for the development of probiotics derived fine products. 


\section{Acknowledgements}

This study was partially supported by grants from the Henan Agency of Science and Technology (grant No. 172102410055, 172102110143). The funders had no role in the study design, data collection and analysis, or decision to publish.

\section{References}

[1] Sun, Z. (2014) Development of Gene Expression Systems in Bifidobacterium bifidum S17 and Their Application for Tumor Therapy. Dissertation, Open Access Repositorium der Universität Ulm, Ulm.

[2] Bazanella, M., Maier, T.V., Clavel, T., et al. (2017) Randomized Controlled Trial on the Impact of Early-Life Intervention with Bifidobacteria on the Healthy Infant Fecal Microbiota and Metabolome. American Journal of Clinical Nutrition, 106, 1274-1286.

[3] Grimm, V., Westermann, C. and Riedel, C.U. (2014) Bifidobacteria-Host Interactions-An Update on Colonisation Factors. BioMed Research International, 2014, 10. https://doi.org/10.1155/2014/960826

[4] Sanchez, B., Urdaci, M.C. and Margolles, A. (2010) Extracellular Proteins Secreted by Probiotic Bacteria as Mediators of Effects That Promote Mucosa-Bacteria Interactions. Microbiology, 156, 3232-3242. https://doi.org/10.1099/mic.0.044057-0

[5] Guglielmetti, S., Mora, D., Gschwender, M., et al. (2011) Randomised Clinical Trial: Bifidobacterium bifidum MIMBb75 Significantly Alleviates Irritable Bowel Syndrome and Improves Quality of Life-A Double-Blind, Placebo-Controlled Study. Alimentary Pharmacology \& Therapeutics, 33, 1123-1132. https://doi.org/10.1111/j.1365-2036.2011.04633.x

[6] Cronin, M., Morrissey, D., Rajendran, S., et al. (2010) Orally Administered Bifidobacteria as Vehicles for Delivery of Agents to Systemic Tumors. Molecular Therapy, 18, 1397-1407. https://doi.org/10.1038/mt.2010.59

[7] Sulek, K., Vigsnaes, L.K., Schmidt, L.R., et al. (2014) A Combined Metabolomic and Phylogenetic Study Reveals Putatively Prebiotic Effects of High Molecular Weight Arabino-Oligosaccharides When Assessed by in Vitro Fermentation in Bacterial Communities Derived from Humans. Anaerobe, 28, 68-77. https://doi.org/10.1016/j.anaerobe.2014.05.007

[8] Hong, Y.S., Hong, K.S., Park, M.H., et al. (2011) Metabonomic Understanding of Probiotic Effects in Humans with Irritable Bowel Syndrome. Journal of Clinical Gastroenterology, 45, 415-525. https://doi.org/10.1097/MCG.0b013e318207f76c

[9] Russell, D.A., Ross, R.P., Fitzgerald, G.F., et al. (2011) Metabolic Activities and Probiotic Potential of Bifidobacteria. International Journal of Food Microbiology, 149, 88-105. https://doi.org/10.1016/j.ijfoodmicro.2011.06.003

[10] O'Shea, E.F., Cotter, P.D., Stanton, C., et al. (2012) Production of Bioactive Substances by Intestinal Bacteria as a Basis for Explaining Probiotic Mechanisms: Bacteriocins and Conjugated Linoleic Acid. International Journal of Food Microbiolo$g y, 152,189-205$. https://doi.org/10.1016/j.ijfoodmicro.2011.05.025

[11] Gorissen, L., De Vuyst, L., Raes, K., et al. (2012) Conjugated Linoleic and Linolenic Acid Production Kinetics by Bifidobacteria Differ among Strains. International Journal of Food Microbiology, 155, 234-240. https://doi.org/10.1016/j.ijfoodmicro.2012.02.012

[12] Martinez, F.A., Balciunas, E.M., Converti, A., et al. (2013) Bacteriocin Production by Bifidobacterium spp. A Review. Biotechnology Advances, 31, 482-488. 
https://doi.org/10.1016/j.biotechadv.2013.01.010

[13] Prosser, G.A., Larrouy-Maumus, G. and de Carvalho, L.P. (2014) Metabolomic Strategies for the Identification of New Enzyme Functions and Metabolic Pathways. EMBO Reports, 15, 657-669. https://doi.org/10.15252/embr.201338283

[14] Gowda, G.A. and Djukovic, D. (2014) Overview of Mass Spectrometry-Based Metabolomics: Opportunities and Challenges. Methods in Molecular Biology, 1198, 3-12. https://doi.org/10.1007/978-1-4939-1258-2_1

[15] Respondek, F., Gerard, P., Bossis, M., et al. (2013) Short-Chain Fructo-Oligosaccharides Modulate Intestinal Microbiota and Metabolic Parameters of Humanized Gnotobiotic Diet Induced Obesity Mice. PLoS One, 8, e71026.

https://doi.org/10.1371/journal.pone.0071026

[16] Turroni, F., Milani, C., Duranti, S., et al. (2016) Deciphering Bifidobacterial-Mediated Metabolic Interactions and Their Impact on Gut Microbiota by a Multi-Omics Approach. ISME Journal, 10, 1656-1668. https://doi.org/10.1038/ismej.2015.236

[17] Sun, Z., Baur, A., Zhurina, D., et al. (2012) Accessing the Inaccessible: Molecular Tools for Bifidobacteria. Applied and Environmental Microbiology, 78, 5035-5042. https://doi.org/10.1128/AEM.00551-12

[18] Osswald, A., Sun, Z., Grimm, V., et al. (2015) Three-Dimensional Tumor Spheroids for in Vitro Analysis of Bacteria as Gene Delivery Vectors in Tumor Therapy. Microbial Cell Factories, 14, 199. https://doi.org/10.1186/s12934-015-0383-5

[19] Sun, Z., Westermann, C., Yuan, Y., et al. (2014) Characterization of a Gap Promoter for High Level Gene Expression in Bifidobacterium bifidum S17. Bioengineered, 5, 371-377. https://doi.org/10.4161/bioe.34423

[20] Osswald, A., Westermann, C., Sun, Z., et al. (2015) A Phytase-Based Reporter System for Identification of Functional Secretion Signals in Bifidobacteria. PLoS ONE, 10, e0128802. https://doi.org/10.1371/journal.pone.0128802 